\title{
ALL-OPTICAL WAVELENGTH ENCODED NAND AND NOR OPERATIONS EXPLOITING SEMICONDUCTOR OPTICAL AMPLIFIER BASED MACH-ZEHNDER INTERFEROMETER WAVELENGTH CONVERTER AND PHASE CONJUGATION SYSTEM
}

\author{
BIJAN GHOSH*, ${ }^{*}$ and SOURANGSHU MUKHOPADHYAY ${ }^{\dagger, \S}$ \\ *Vidyasagar University, Department of Physics and Technophysics \\ Midnapur, West Bengal, India, PIN-721102 \\ ${ }^{\dagger}$ The University of Burdwan, Golapbag \\ Burdwan, India, PIN-713104 \\ ¥bijanghosh_5@yahoo.co.in \\ §sourangshu2006@hotmail.com
}

Received 1 June 2011

\begin{abstract}
Non-linear materials have already been established as optical switch, which is the gateway for implementation of all-optical logic gates. Semiconductor optical amplifier (SOA), optical phase conjugation system (PCS) and Mach-Zehnder Interferometer (MZI) have also been used several times for implementing all-optical gates. Here in this paper, the authors propose a new scheme for implementing optical NAND and NOR logic gates (which are universal gate also) by the application of the wavelength encoding principle, which has a lot of advantages over the conventional intensity and polarization based encoding principle.
\end{abstract}

Keywords: Semiconductor optical amplifiers; MZI wavelength converter; optical phase conjugation system; optical logic gate; NAND and NOR.

\section{Introduction}

To develop a super fast optical computer, scientists are massively involved with the development of all-optical logic gates. Researches in the area of optical computing are also engaged to develop several new optoelectronic and all-optical combinational as well as sequential logic processors ${ }^{3-8}$ with these gates such as optical flip-flops, optical memory units, optical counters etc. Several types of optical logic gates have been proposed and implemented successfully in the past few decades.

In this context, optical Fredkin gates, logic gates by massive use of non-linear photorefractive material based systems, space variant logic gates etc. can be mentioned. Semiconductor optical amplifiers (SOAs) are also a very strong candidate for implementing various switching operations and logic gates. The response bit rate of these amplifiers is higher than $100 \mathrm{~Gb} / \mathrm{s}$. Again SOA needs a mw level or threshold power for its operation. 
The semiconductor optical amplifiers can be used for implementing several active optical functional applications, such as optical switches, pulse generator, wavelength converter etc. Wavelength conversion can be done by the exploitation of the cross gain modulation (XGM) character of SOA, which is based on intensity-modulated optical gain variation character of SOA placed in Mach-Zehnder configuration with XPolM in the SOA. ${ }^{10,11}$ SOA has already been proposed for use in all-optical ultra high speed half-adder also. ${ }^{12}$

In this paper, we propose a promising approach for the method of implementation of an all-optical wavelength encoded NAND operation with joint uses of non-linear material (NLM) and semiconductor optical amplifier. Here we use the properties of NLM based phase conjugation system and semiconductor optical amplifier (SOA) based Mach-Zehnder Interferometer (MZI) as wavelength converters. In SOA, electrical pumping mechanism is used for the conversion of one optical signal at a specific wavelength to another one of different wavelength. This optical network functions well in the wavelength range (15581570) $\mathrm{nm}$ and with channel spacing of $200 \mathrm{~Hz}$, and it can give us a high speed all-optical communication system for supporting wavelength division multiplexing. The switching time is of the order of $100 \mathrm{ps}$.

\section{Optical NLM Based PCS as Optical Switch}

The optical non-linear material (OPNLM) based phase conjugation system (PCS) by the use of probe and pump beams in non-linear material is shown in Fig. 1. Two pump beams and one probe beam are incident in this medium, and point and form a real time grating at the interference. The total field can be expressed as

$$
\mathrm{E}(t) \infty^{1 / 2}\left[\mathrm{E}_{f}+\mathrm{E}_{b}+\mathrm{E}_{p}+\text { Complex Conjugate }\right]^{3}
$$

$\mathrm{E}_{f}$ and $\mathrm{E}_{b}$ are fields of forward and backward pump beams respectively. The power of the probe beam is $\mathrm{E}_{p}$.

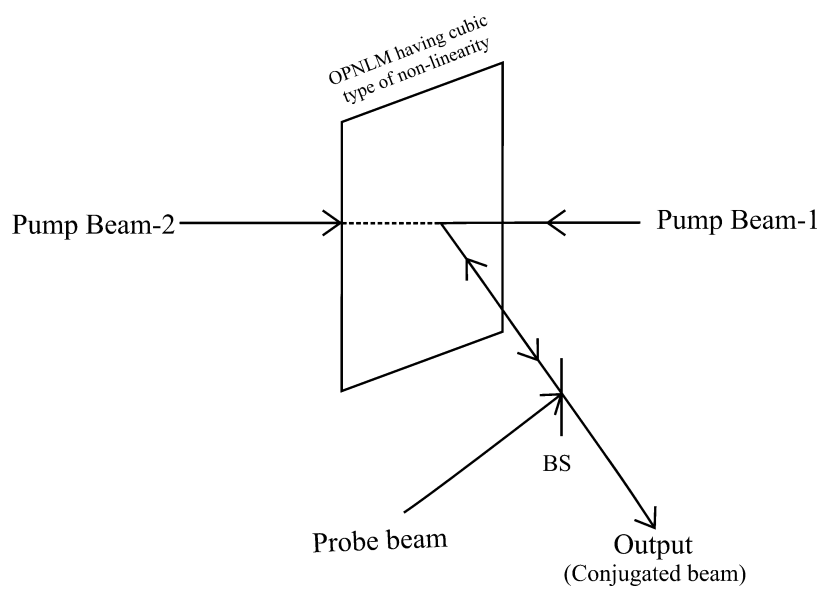

Fig. 1. OPNLM based PCS used as switch. 
The switching mechanism can be discussed as follows. When two input pump beams excite the NLM, a probe beam is seen to be reflected from itself and when any one or both the input beams are dissimilar or absent, the probe beam is not allowed to be reflected from it. As the phase conjugation occurs when both the pump beam and the probe beam are of the same wavelength and polarization, so one can obtain the phase conjugated output at the wavelength $\lambda_{1}$ when all the probe and pump beams are of wavelength $\lambda_{1}$. Therefore the scheme can be thought of as an all-optical switch.

It is already seen that a point in the NLM is sufficient to organize the phase conjugation, so the dimension of the switch is very small, i.e. in the order of beam dimension. Thus the all switch dimension is in sub micron level. A NLM of $1 \mathrm{~cm}^{2}$ dimension can perform thousands of phase conjugation if the exiting laser is of $1 \mu \mathrm{m}^{2}$ dimension. If proper non-linear material is identified, where the 2 nd order non-linear coefficient is sufficiently high, then $\mu \mathrm{W}$ to $\mathrm{mW}$ level of optical power requires a small optical power to be used as an optical switch.

\section{SOA Based MZIWC used also as Optical Switch}

Cross gain modulation (XGM) is one of the main characters of SOA nonlinearity. Carrier density changes in SOA material due to homogenous broadening of material gain spectrum. This change in carrier density will affect all of the input signals. Therefore a strong signal at one wavelength is seen to affect the gain of a weak signal at another wavelength. This mechanism is called XGM. ${ }^{13-15}$ Here, two signals are injected into SOA placed in interferometer configuration (MZI.SOA configuration) to convert phase changes in the signals to intensity changes using destructive and constructive interference. The configuration is in asymmetric MZI wavelength converting mode shown in Fig. 2. One can obtain the intensity modulated $\lambda_{2}$ at the output for the application of the intensity modulated $\lambda_{1}$, along with a continuous wavelength which is $\lambda_{2} \cdot 9,16$

The main advantage of SOA is that all four types of non-linear operations (cross gain modulation, cross phase modulation, wavelength conversion and four wave mixing) can be conducted. Again, SOA is cost effective and can be run with a low power laser.

The disadvantages of SOA is that the electrical pumping power is very much essential for activating the SOA system. For these operations, a speed of $100 \mathrm{THz}$ is difficult to achieve.

\section{Method of Implementing All-Optical NAND with MZIWC and PCS}

We choose a phase conjugation system which is a laser based non-linear operation with probe and pump beams. Only when both inputs of the same frequency (as well as wavelength)

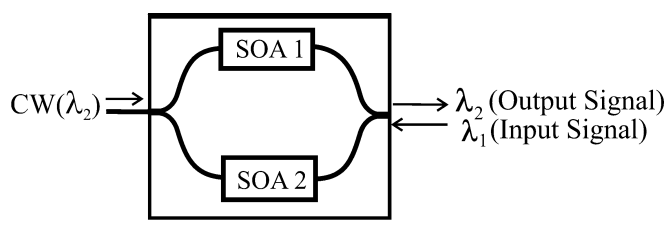

Fig. 2. Asymmetric MZI wavelength conversion scheme. 


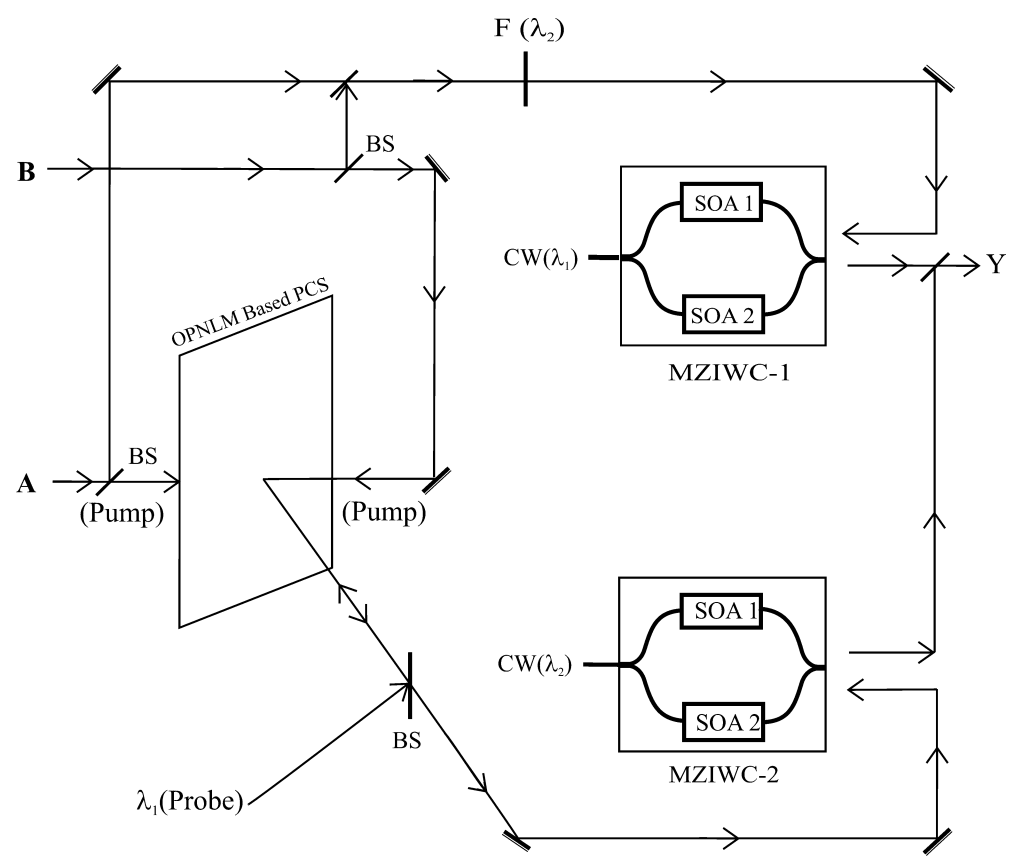

Fig. 3. NAND operation with asymmetric MZIWC and PCS.

beam (from A and B as shown in Fig. 3) excites the NLM, then the probe beam of wavelength $\left(\lambda_{1}\right)$ is seen to be reflected from the material and the light of wavelength $\lambda_{1}$ is obtained at the output. When two inputs are dissimilar, the NLM will resist the probe beam for the above reflection. ${ }^{1,2}$ This type of NLM takes on an important role for implementing the NAND and NOR operations. The speed of this NLM based PCS switch can show the real time operation. This PCS performs the AND operations in both the NAND and NOR logic schemes. The PCS and SOA jointly serve the respective NAND and NOR operations. Again, a number of operations can be conducted by optical NLM based PCS. So the parallelism in NLM ultimately enhances the speed of operation of the whole scheme.

To implement the NAND operation, we require two asymmetric MZI systems and one phase conjugation system shown in Fig. 3. In one asymmetric MZI wavelength conversion system (MZIWC-1), the continuous wave (CW) input is $\lambda_{1}$. Only when the input $\lambda_{2}$ is applied to it, will the converted output $\lambda_{1}$ be obtained. Similarly in MZIWC-2, the CW input is $\lambda_{2}$ and thus only when the input signal $\lambda_{1}$ is applied to it, will we receive the $\lambda_{2}$, which is received from the output terminal of the MZIWC-2. The optical circuit diagram of the whole NAND system is shown in Fig. 3. Before the description of the whole operation, it should be stated that $\lambda_{2}$ wavelength is coded as 0 bit and $\lambda_{1}$ is coded as 1 bit in the Boolean mechanism. Considering this coding, we have the truth table of NAND logic given in Table 1.

Here $\lambda_{1}$ and $\lambda_{2}$ are light of the same polarization. We treat $\lambda_{1}$ as high state i.e. 1 and $\lambda_{2}$ as low state i.e. 0. A and B are the input terminals of the NAND whereas $\mathrm{Y}$ is the output terminal of the same. 
Table 1. Truth table of wavelength encoded NAND logic.

\begin{tabular}{lcc}
\hline A & B & Y \\
\hline$\lambda_{2}(0)$ & $\lambda_{2}(0)$ & $\lambda_{1}(1)$ \\
$\lambda_{2}(0)$ & $\lambda_{1}(1)$ & $\lambda_{1}(1)$ \\
$\lambda_{1}(1)$ & $\lambda_{2}(0)$ & $\lambda_{1}(1)$ \\
$\lambda_{1}(1)$ & $\lambda_{1}(1)$ & $\lambda_{2}(0)$ \\
\hline
\end{tabular}

When $\mathrm{A}=\lambda_{2}(0)$ and $\mathrm{B}=\lambda_{2}(0)$, no light is reflected from the phase conjugation system (PCS). However, F filter passes the light of wavelength $\lambda_{2}$, and we get $\lambda_{1}(1)$ from MZIWC-1. Therefore, the output is $\lambda_{1}(1)$.

Secondly, for $\mathrm{A}=\lambda_{2}(0)$ and $\mathrm{B}=\lambda_{1}(1)$, the $\mathrm{F}$ filter passes the light of wavelength $\lambda_{2}$ and we receive output from Y through MZIWC-1 as $\lambda_{1}(1)$. Like the earlier case here, the phase conjugation system also does not work due to dissimilar pump and probe beams.

Now when $\mathrm{A}=\lambda_{1}(1)$ and $\mathrm{B}=\lambda_{2}(0)$, the phase conjugation system will also not work. F selects only $\lambda_{2}$ through it, which produces $\lambda_{1}(1)$ at the output.

Finally, when $\mathrm{A}=\lambda_{1}(1)$ and $\mathrm{B}=\lambda_{1}(1)$, the PCS is activated and the probe beam $\lambda_{1}$ will be reflected from the PCS, and this light of wavelength $\lambda_{1}$ comes to MZIWC-2 block (whose CW input is $\lambda_{2}$ ) as an input. So one can receive the $\lambda_{2}(0)$ output from this block, which is connected to Y.

Thus when $\mathrm{A}$ and $\mathrm{B}$ are in the same high state i.e. $\mathrm{A}=\lambda_{1}(1)$ and $\mathrm{B}=\lambda_{1}(1)$, then MZIWC-2 and OPNLM based PCS function and respective output is received at port Y. In the same way for all the other combinations, OPNLM based PCS does not work to supply its signal to MZIWC-2, as its character is already described, and only MZIWC-1 functions accordingly to provide the respective output at port Y.

The system is simple in design and fabrication. (The simplicity and ease of fabrication are also exploited in the implementation system as per possible of NOR gate, with the same methodology.) In Fig. 4, we have shown the extension of the scheme for developing a logical system having a large number of wavelength encoded inputs. $Y_{n}$ is the number of different outputs that can be received from the integrated all-optical systems.

\section{Method of Implementing All-Optical NOR with MZIWC and PCS}

Now the PCS and MZIWC (SOA based) can be jointly used to organize the all-optical NOR operation also. It is shown in Fig. 4. The basic block diagram of NOR operation is constructed with the help of one PCS and two SOA-MZIWC switches (MZIWC-1 and MZIWC-2). One pass filter $\mathrm{F}\left(\lambda_{1}\right)$, four optical beam splitters and seven mirrors are used for designing the circuit for implementation. We consider this coding to have the truth table of NOR logic as given in Table 2.

When $\mathrm{A}=\lambda_{2}(0)$ and $\mathrm{B}=\lambda_{2}(0)$, the PCS is activated and the probe beam $\lambda_{2}$ will be reflected from the PCS. The light of wavelength $\lambda_{1}$ comes to MZIWC-2 block (whose CW 


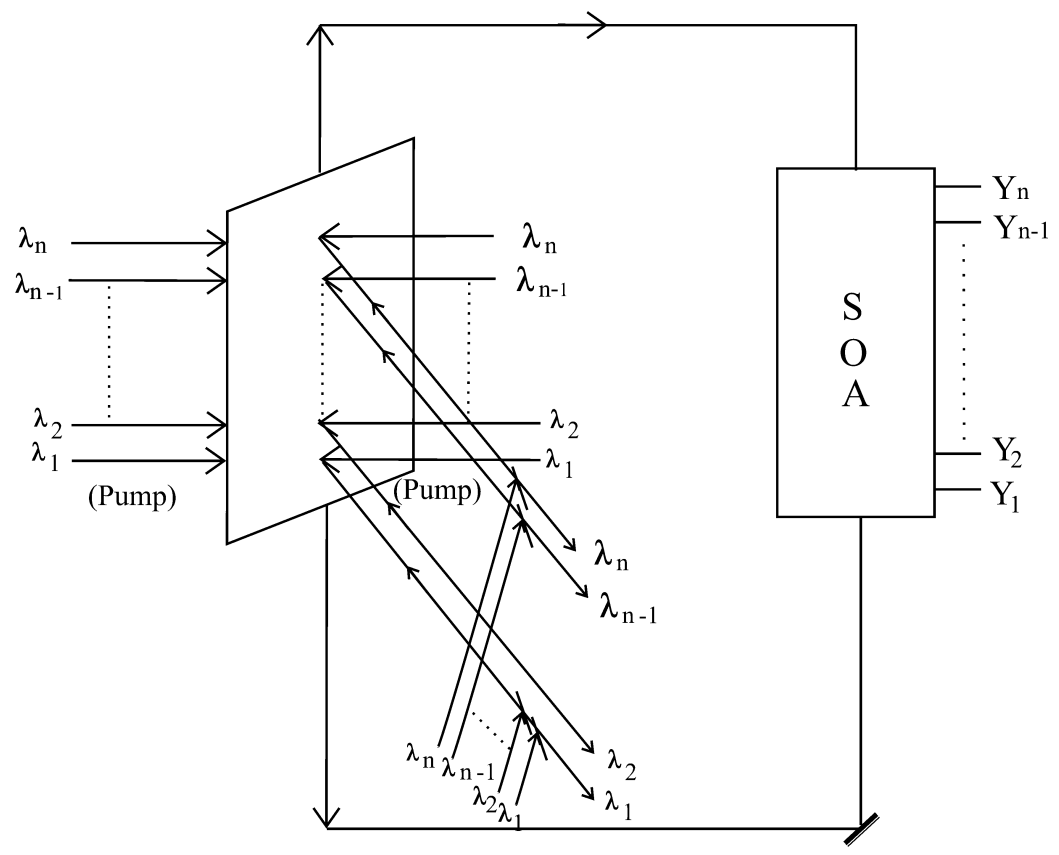

Fig. 4. Integrated all-optical system.

Table 2. Truth table of wavelength encoded NOR logic.

\begin{tabular}{lcc}
\hline A & B & Y \\
\hline$\lambda_{2}(0)$ & $\lambda_{2}(0)$ & $\lambda_{1}(1)$ \\
$\lambda_{2}(0)$ & $\lambda_{1}(1)$ & $\lambda_{2}(0)$ \\
$\lambda_{1}(1)$ & $\lambda_{2}(0)$ & $\lambda_{2}(0)$ \\
$\lambda_{1}(1)$ & $\lambda_{1}(1)$ & $\lambda_{2}(0)$ \\
\hline
\end{tabular}

input is $\left.\lambda_{1}\right)$ as an input. Therefore one can receive the $\lambda_{1}(1)$ output from this block, which is connected to $\mathrm{Y}$.

Secondly, for the next two cases $\left[\mathrm{A}=\lambda_{2}(0)\right.$ and $\left.\mathrm{B}=\lambda_{1}(1)\right]$ and $\left[\mathrm{A}=\lambda_{1}(1)\right.$ and $\left.\mathrm{B}=\lambda_{2}(0)\right]$, the phase conjugation system will not work due to dissimilar pump and probe beams. The filter $\mathrm{F}$ passes the light of wavelength $\lambda_{1}$, and we receive the output from $\mathrm{Y}$ through MZIWC-1 (whose CW input is $\lambda_{2}$ ), which is $\lambda_{2}(0)$.

Finally when $\mathrm{A}=\lambda_{1}(1)$ and $\mathrm{B}=\lambda_{1}(1)$, no light is reflected from the phase conjugation system (PCS) for dissimilarity in pump beam $\left(\lambda_{2}\right)$ and two probe beams. The filter F passes the light of wavelength $\lambda_{1}$, and we receive the output from Y through MZIWC-1 (whose CW input is $\left.\lambda_{2}\right)$, which is $\lambda_{2}(0)$ as in the earlier two cases.

Thus when $\mathrm{A}$ and $\mathrm{B}$ are both in the low state i.e. $\mathrm{A}=\lambda_{2}(0)$ and $\mathrm{B}=\lambda_{2}(0)$, then MZIWC-2 and OPNLM based PCS function and respective output are received at port Y. In the same way, for all the other combinations, OPNLM based PCS does not work to 


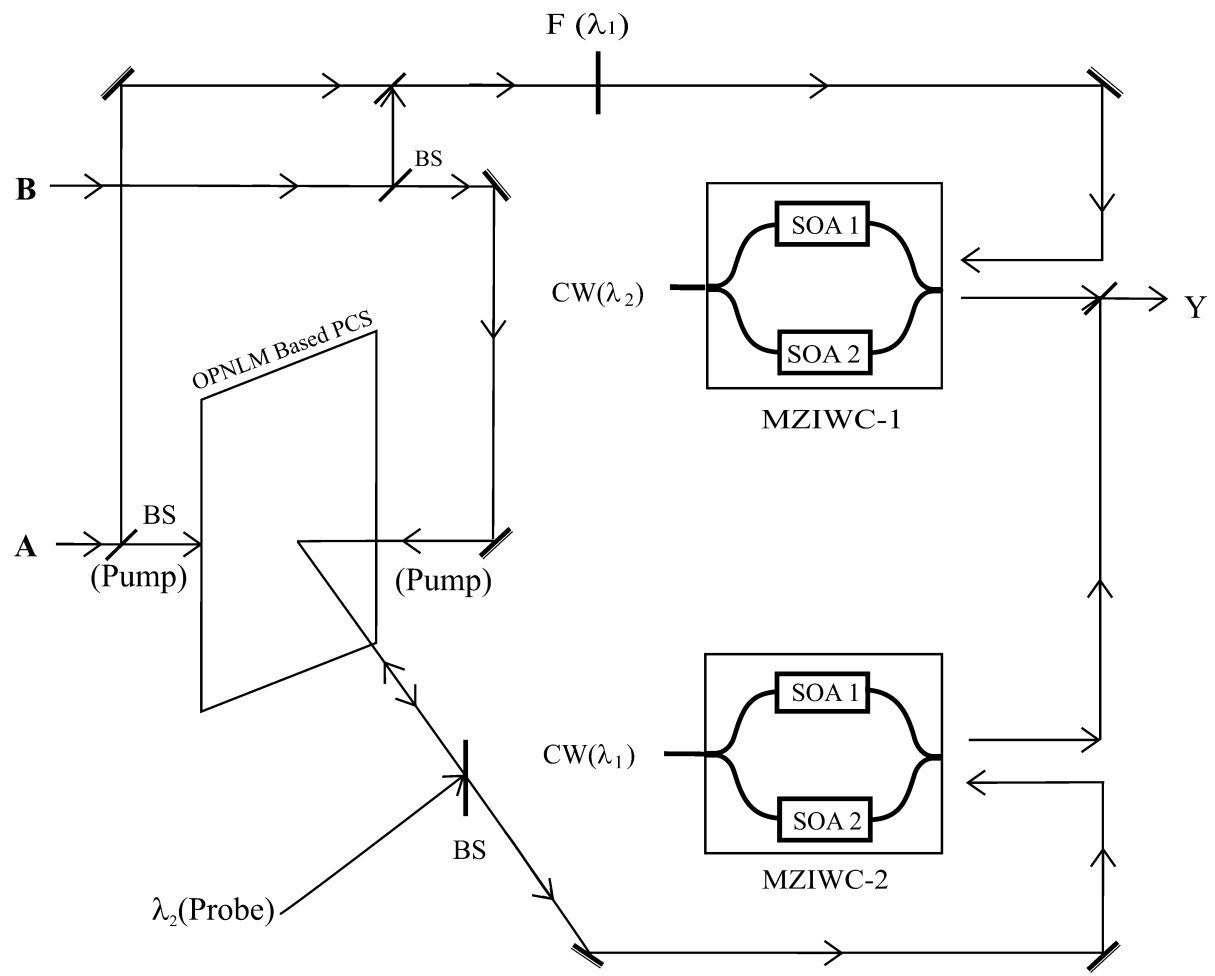

Fig. 5. NOR operation with asymmetric MZIWC and PCS.

supply the signal to MZIWC-2 as per its character already described, with only MZIWC-1 functioning accordingly to provide the respective output at port Y.

\section{Conclusion}

An all-optical NAND operation with asymmetric MZI-wavelength converter can be realized by XPolM at a bit rate of $2.5 \mathrm{Gbit} / \mathrm{s}$. SOA technology is capable of realizing many of the all-optical applications, but we have to remember that the input signal power and internal noise (7-12) dBm, which is the cause of gain of SOA, may be the cause of signal distortion at saturation stage. Here it is reported that we have developed the NAND logic gate as NAND is the universal logic one. If NAND is implemented, one can implement any other logic gate. Logic gates with SOA have already been developed and proposed by many researchers. Here, the sufficient difference of the proposed mechanism from others is the wavelength encoded implementation of logic operation, as wavelength encoding has many advantages over intensity based and polarization based conventional encoding principles.

The advantage of this scheme over the other schemes is that the frequency encoded logic operation is conducted with less system complexity and with high speed over the conventional methods. The wavelength conversion is done by SOA, and the speed of operation of 
each scheme is increased by the parallelism of NLM based PCS. One can convert NAND logic to NOR logic by replacing the pass band filter $\mathrm{F}\left(\lambda_{2}\right)$ by $\mathrm{F}\left(\lambda_{1}\right)$ and the probe beam $\lambda_{1}$ by $\lambda_{2}$.

Again, the limitation of the scheme is the comparatively lower $(100 \mathrm{THz})$ speed of operation of SOA over the connectional all optical switches. In spite of this limitation, one cannot avoid SOA because of its wavelength conversion activity.

It is interesting to see that if optical pulses are used instead of analog optical probe and pump beams, one can achieve high optical pulse power with small energy consumption. If the duration of the pulses is shortened, the power of the pulses will increase. Therefore due to an increase of pulsating input power, the non-linearity of the NLM can be easily activated. Here, the optical switching will be more comfortable when pulsating inputs are used instead of CW inputs.

As the thickness of both the NLM and the SOA are very small (order of mm or less than that), therefore the dispersion issue will be negligible. Similarly, if the thickness increases, the question of dispersion will automatically come. To implement the operation, we do not require the increase of thickness. This increase will not only contribute to dispersion, but also reduce the conjugation efficiency and extinction coefficient, which hamper the logic operation.

\section{References}

1. S. Mukhopadhyay and J. N. Roy, A minimization scheme of optical space-variant logical operations in a combination architecture, Opt. Comm. 119 (1995) 499-504.

2. Jazbinsek, M. Bach, T. Montemezzani, G. Gunter, P. Grabar, A. A. Vysochanskii and Yu. M., Inst. of Quantum Electron., Swiss Fed. Inst. of Technol., Zurich, Switzerland, Optical phase conjugators for near-infrared diode lasers using $\mathrm{Sn}_{2} \mathrm{P}_{2} \mathrm{~S}_{6}$ crystals, Lasers and Electro-Optics, 2005. (CLEO). Conference. 10.1109/CLEO.2005.202216.

3. T. Yatagi, Optical space-variant logic-gate array based on spatial encoding technique, Opt. Lett. 11(40) (1986) 260-262.

4. J. Tanida and Y. Ichioka, Optical logic array processor using shadowgrams, J. Opt. Soc. Am. A 73 (1983) 800.

5. K. R. Chowdhury and S. Mukhopadhayay, Binary optical arithmetic operation scheme with tree architecture by proper accommodation of optical non-linear materials, Opt. Eng. 43(1) (2004) $132-136$.

6. Bijan Ghosh, Radha Raman Pal and S. Mukhopadhyay, An all-optical method of binary data multiplication with use of the Kerr nonlinearity, The IUP Journal of Physics 3(2) (2010) 29-35.

7. Bijan Ghosh, Radha Raman Pal and Sourangshu Mukhopadhyay, An all-optical integrated system for implementing arithmetic operation in 2's complement method with the active participation of non-linear material based switches, Indian J. Phys. 84(8) (2010) 1101-1109.

8. S. Mukhopadhyay, A. Basuray and A. K. Dutta, A new technique of arithmetic operation using position residue system, Appl. Opt. 29 (1990) 20.

9. N. K. Datta and Q. Wang, Semiconductor Optical Amplifiers (World Scientific Publishing, Singapore, 2006).

10. L. Q. Guo and M. J. Connelly, Signal-induced birefringence and dichroism in a tensile-strained bulk semiconductor optical amplifier and its application to wavelength conversion, J. Lightwave Technol. 23 (2005) 4037-4045. 
11. L. Q. Guo and M. J. Connelly, Demonstration of birefringence in a bulk semiconductor optical amplifier and its application to wavelength conversion, in Technical Digest: Symposium on Optical Fiber Measurements 2004, P. A. Williams and G. W. Day, eds. NIST, (Boulder, Colo., 2004), pp. 167-170.

12. Pei-Li Li, De-Xiu Huang, Xin-Liang Zhang and Guang-Xi Zhu, Ultrahigh-speed all-optical half adder based on four-wave mixing in semiconductor optical amplifier, Opt. Express 14 (2006) 11839-11847.

13. L. Q. Guo, All-optical wavelength conversion and logic gate using nonlinear polarization rotation in a semiconductor optical amplifier, $\mathrm{Ph}$. D dissertation. University of Limerick, Ireland, 2006 (Chapters $2 \& 4)$.

14. Bijan Ghosh, Radha Raman Pal and Sourangshu Mukhopadhyay, A new approach to all-optical half-adder by utilizing semiconductor optical amplifier based MZI wavelength converter, OptikInt. J. Light Electron Opt. (2010), doi:10.1016/j.ijleo.2010.10.042.

15. M. Asghari, I. II. White and R. V. Penty, Wavelength conversion using semiconductor optical amplifiers, J. Lightwave Technol. 15(7) (1997) 1181-1190.

16. Michael J. Connelly, Semiconductor Optical Amplifiers (Kluwer Academic Publishers, Boston, Netherlands, 2002). 